\title{
Creativity in the workplace
}

\author{
By Anne T. Ostrye \\ Assistant Director for Information Services \\ University of Wyoming Libraries
}

\section{A report on the ACRL President's Program in New Orleans.}

$\mathbf{T}$ The ACRL President's Program at the 107th ALA Annual Conference presented "Creativity in the Workplace: From Conception to Application." The 400 participants were held spell bound in a creative atmosphere for the $2^{1 / 2}$ hour program.

Scott G. Isaksen from the Center for Studies in Creativity, State University College at Buffalo, discussed basic concepts, theories and current research on creativity. Susan Jurow, associate director of the Association of Research Libraries, Office of Management Services, followed Isaksen and presented practical techniques to foster creative problem-solving in the workplace.

According to Isaksen, creativity is seen by most people as so mysterious that it cannot be studied; as so magical that only a few gifted souls are really creative; or as somehow linked to madness - the creative individual is perceived as weird or abnormal. Creativity, however, has been subject to nearly forty years of scientific inquiry, research, and writing, and Isaksen presented the results of several of these studies.

Further, he views creativity as a multifaceted phenomenon with numerous definitions. But these definitions have one common thread: creativity is "novelty that is useful." That is, the process of creativity must produce something that is useful. His formula for the process is $\mathrm{CF}(\mathrm{K}, \mathrm{I}, \mathrm{E})$. That is, the function ( $f$ ) of creativity $(\mathrm{C})$ is knowledge $(\mathrm{K})$, imagination (I), evaluation (E). It is an ongoing, dynamic concept.
Isaksen brought the concepts of creativity down to earth for the audience by describing four related elements of creativity as outlined by Rhodes in 1961: 1) Personality, or, the traits, characteristics or attributes of the creative personality must be understood. 2) Process, the stages of thinking creative people use to invent a new, useful product, must be described. 3) Product, the qualities of the product which make it both novel useful must be understood. 4) The nature of an environment conducive to creative thinking must be understood.

He then discussed the work of Torrance on the creative personality done in the 1950 s. Torrance studied the cognitive and affective characteristics of highly creative people, including fluency, flexibility, originality, elaboration, curiosity, complexity, risk-taking and imagination.

The model Torrance uses for predicting highlevel creative behavior goes beyond identifying specific characteristics. Instead, he considers three broad categories of personality including abilities (things like intelligence), motivations and skills. His conclusion is that if you want to predict high levels of creative behavior it is important to have reasonably high levels of all three of these categories. Having an extremely high level of one would not be as productive as having an above average level of all three.

Isaksen continued demystifying creativity by describing four stages in the creative process: 1) preparation, (investigating the problem in all direc- 
tions), 2) incubation (thinking about the problem in a "not conscious" manner), 3) illumination (the appearance of a "happy idea"), and 4) verification (making it a reality and reducing the idea to an exact form). Preparation and verification are the stages that connect with the outside world, helping a person deal with reality and make the creative product useful. The two other stages, incubation and illumination, are internal processes. These stages make products novel or creative. By understanding the creative process, a person can develop creative problem-solving techniques, become more imaginative, and thus become more effective in both thinking and action.

The creative product is really the basis on which all research on creativity rests. A creative product is new and it is useful, relevant and transferable. Again, to be creative, the product must have "novelty that is useful."

Finally, the environment must be conducive to creative problem-solving. Isaksen pointed out that in most organizations novelty is disconcerting and hard to manage in a group setting. However, an atmosphere favorable to creativity fosters innovation and creative problem-solving. Based on work conducted by Ekvall at the Swedish Council for Management and Work Life Issues, the Center for Studies in Creativity has identified ten components that shape an environment conducive to creativity and innovation:

1) Challenge. A high-challenge climate results when the people are experiencing joy and meaningfulness in their work, and therefore, they invest much energy.

2) Freedom. In a climate that allows independence in behavior, people make contacts to give and receive information. They discuss problems and alternatives. They plan and take initiatives of different kinds. They can make decisions.

3) Idea Support. In a supportive climate, ideas and suggestions are received in an attentive and kind way by supervisors and co-workers. People listen to each other and encourage initiative. The atmosphere is constructive and positive.

4) Trust/Openness. When there is a strong level of trust, everyone in the organization dares to put forward ideas and opinions. Initiatives can be taken without fear of reprisal or ridicule in case of failure. Communication is open and straight forward.

5) Dynamism/Liveliness. In a highly dynamic situation, new things are happening all the time and alternatives between ways of thinking about and handling issues often arise. There is a kind of psychological turbulence which is described by people in those organizations as "full speed," "go," "breakneck," "maelstrom," and the like.

6) Playfulness/Humor. A relaxed atmosphere with jokes and laughter characterizes the organization which is high in the creative dimension.

7) Debates. In the debating organization many voices are heard and people are keen on putting forward their ideas.

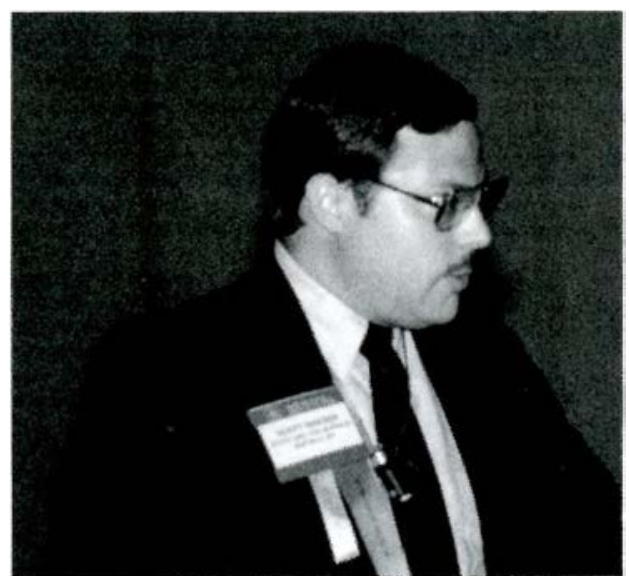

Scott Isaksen

8) Conflicts. When the level of conflict is high, groups and single individuals dislike each other and the climate can be characterized by "warfare." Plots, traps, gossip and slander characterize life in the organization. When conflict in the organization is low, people behave in a mature manner; they have psychological insight and control of impulses.

9) Risk-taking. In an environment that favors taking risks, decisions and actions are prompt and rapid. Opportunities are taken as they arise and action is preferred to detailed investigation and analysis. In a risk-avoiding climate there is a cautious, hesitant mentality. People try to be on the "safe side." They decide to "sleep on the matter." They set up committees and cover themselves in many ways before making a decision.

10) Idea Time. This is the amount of time people can use (and do use) for elaborating new ideas. In the high idea-time situation, it is possible to discuss and test impulses and new suggestions that are not planned or included in the task assignment; people tend to use these possibilities.

With an understanding of the creative personality, the creative process, the product and the need for an atmosphere conducive to creativity, the audience was ready for Susan Jurow to present various techniques that lead to creative problemsolving in their own work situation.

Jurow began by articulating the reason so many librarians were attending a workshop on creativity. Fundamentally, librarians need and want newer and fresher ideas, flexibility in facing new demands, and ideas for new answers to old demands.

In choosing techniques to foster new, creative problem-solving, a person needs to decide his or her personal strengths and weaknesses and the kind of resulting product he or she is striving for given the context of the problem.

Jurow observed that there are no simple, quick 
and accurate measurements for different levels of creativity. There are, however, some psychometric instruments to measure styles of creativity and the styles that a person may naturally use. She described the Kirton Adaption-Innovation Inventory for the audience. Michael Kirton theorized that the individual style of creativity is related to the way an individual tends to define problems and make decisions. People tend to prefer either an adaptive approach or an innovative approach. Jurow defined these approaches and asked the audience to examine what approach they prefer to use in daily work.

Adaptors are disciplined in their problemsolving, working within the boundaries of a problem as it is defined. For example, if asked to study how the library could bind periodicals in a more cost-effective manner, adaptors would study the fee structure of different binders, explore the costs of in-house binding, and examine the feasibility of microform replacement. The adaptor's contribution is often viewed in terms of their precision, reliability and dependability.

Innovators see themselves and are often seen by others as undisciplined when it comes to problemsolving. They think tangentially, approaching problems from seemingly odd angles. They challenge problem definitions and question the assumptions that others have made about problems. In the above example of binding, the innovator would question the need to bind the journals and even could go so far as to question the need to subscribe to them at all. Instead, their solution might be to contract out for a service to provide instant fulfillment of journal article requests by telefax from a central location. Innovators are perceived as insightful and ingenious because in their search outside the bounds of the problem definition, they find unique approaches. As Isaksen noted, innovators make co-workers and supervisors uncomfortable because their ideas may seem too farfetched or too unfeasible.

Jurow noted that most individuals operate in the middle range using some of each style with a slight inclination towards one over the other. These "middle" people are the ones who keep communication open between the innovators and adaptors and who act as intermediaries or interpreters, helping the innovators and adaptors accept a particular idea. When asked for a show of hands for adaptors, innovators, and those who fall in the middle, the vast majority of the audience fell in the middle as communicators and arbitrators.

Understanding individual styles can give some insight into the dynamics of interactions with others in problem-solving situations. Understanding other styles and how they operate provides an appreciation and ability to use different approaches constructively.

Moving from this end of the spectrum, Jurow connected individual style to four problem-solving approaches: direct, supplementary, modification and tangential. Adaptors tend to use the direct or supplementary approaches in problem-solving. They are likely to simply substitute one solution for another similar one. In solving a security problem in a library, the adaptor would replace the monitor checking books at an exit with an electronic system. This is a direct substitution for the previous solution.

Moving toward the center of the continuum but still near the adaptor side, a supplementary approach would modify the space so that other activities could take place while still alleviating the security problem. For example, a combined checkout/ exit desk.

Moderate innovators "modify" the situation in their problem-solving approach. In the above example, the moderate innovator would eliminate the problem exit and reroute traffic to an exit easier to manage. The extreme innovator would use the tangential approach, question the original problem definition, and propose that security is only a problem when people feel like they have to smuggle things out. If the library permitted unlimited checkout for unlimited periods, security would not be a problem.

Jurow then presented four techniques a librarian could use that would result in creative solutions given the problem and the type of people involved.

Brainstorming is a good technique to use if a person needs a supplementary solution or needs to modify the problem. With brainstorming a person goes for cross-fertilization and quantity, piggybacking on other people's ideas to create new ideas.

Further down the innovative end of the spectrum is a technique called visual connection in which a person moves out of the context of the problem, allowing a myriad of unrelated thoughts, experiences, and observations to bring fresh insight to a problem. This technique was illustrated to the audience by presenting four slides and instructing the audience to relax and imagine being in the picture but also noting how they feel looking at the picture. After writing down impressions the audience was asked to make a connection with the problem statement. Visual connection is one technique that can be done alone; no group interaction is needed. The idea is to clear the mind, concentrate on something unrelated and see what new thoughts have been stimulated regarding the original problem. Innovators often do this naturally. They break the bounds of the problem definition to bring odd angles of thought not immediately obviously relevant to the problem at hand.

The third creative technique presented is called novel scenarios. This technique starts by taking a word out of the problem statement and stringing together word associations. In the problem statement, "How to enhance the role of academic librarians," word associations are made beginning with the word "role" and ending by connecting the words back to the problem statement. No matter how remote, bringing a new perspective to the 


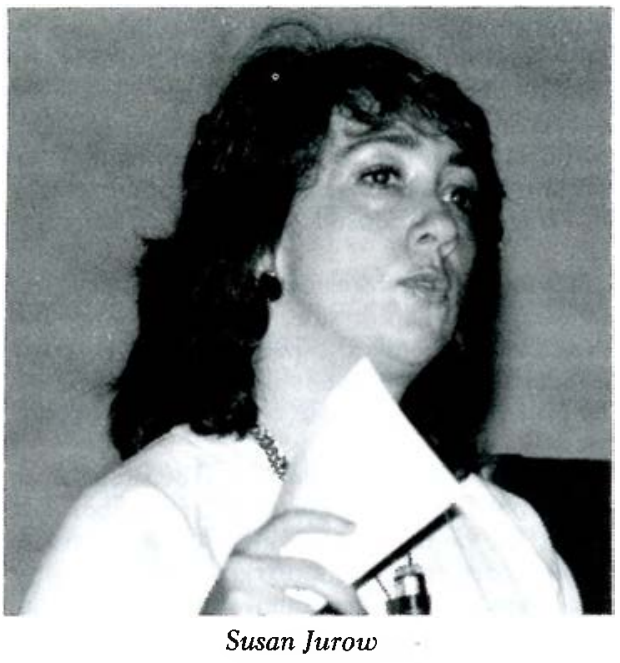

problem is the purpose. A novel scenario, like visual connection, is an excursion technique that allows new perspectives to enter the solution.

At the adaptive end of the spectrum, a useful technique is called brainwriting, which uses the best elements of brainstorming but is more effective for the introverted members of a group who often have trouble getting a word in edgewise. In this technique a problem is stated and each member of the group writes down three solutions. These solutions are exchanged among members of the same group who piggy-back on them creating three more solutions. Each solution creates new associations and thus new ideas. Though this technique is not as energy-intensive as brainstorming, the level of production is fairly impressive. It results in a myriad of fresh ideas from which to choose.

These four techniques, one from the adaptive (brainwriting), two from the innovative (novel scenarios and visual connection) and one from the middle (brainstorming), can be used individually to help jar a person out of routine thinking patterns. They can also be used in a group where they are even more effective. The energy and the diversity of viewpoints increase the group's potential for quantity and variety of ideas. Jurow mentioned that the Office of Management Services at ARL is just starting to develop programs to train librarians based on these techniques.

Concluding her workshop, Jurow reminded the participants to make the best use of what they have learned about creativity techniques: the need to know who you are; the need to understand personal strengths and weaknesses; the need to understand and use the techniques that are available for creative problem-solving; and the need to realize the kind of end product desired.

Jurow returned to Ekvall's ten factors for creating an environment conducive to creativity, emphasizing that a relaxed atmosphere where playfulness and humor are the norm is one of the variables that contributes to a creative organization. She also reminded the audience that the way a manager or supervisor behaves and the messages he or she gives out about these ten factors plays a role in the way staff members feel about an organization and the way they will operate.

The message left with the audience by both Isaksen and Jurow is to take risks and try new ideas. Do not back away from new, exciting approaches to problem-solving because the approaches are too new, too different or too unusual. Finally, participants were urged to use these approaches to creative problem-solving in their daily work.

\section{Bibliography}

Adams, James L. Conceptual Blockbusting, 2nd ed. (New York: W.W. Norton, 1979).

Amabile, Theresa M. The Social Psychology of Creativity (New York: Springer-Verlag, 1983).

Campbell, David P. Take the Road to Creativity and Get Off Your Dead End (Niles, Ill.: Argus Communications, 1977).

de Bono, Edward. Lateral Thinking for Management (New York: McGraw-Hill, 1972).

Drucker, Peter F. Innovation and Entrepreneurship (New York: Harper and Row, 1985).

Ekvall, G., and Andersson, Y. "Working Climate and Creativity: A Study of an Innovative Newspaper Office." Journal of Creative Behavior 20 (1986): 215-225.

Gryskiewicz, Stanley S. "Targeted Innovation." Issues \& Observations, November 1983 (Greensboro, N.C.: Center for Creative Leadership).

Isaksen, Scott G., ed. Frontiers in Creativity Research: Beyond the Basics (Buffalo: Bearly Limited, 1987).

Miller, William C. The Creative Edge (Reading, Mass.: Addison-Wesley, 1987).

Osborn, Alex F. Applied Imagination, 3rd rev. ed. (New York: Charles Scribner's Sons, 1979).

Parnes, Sidney J. The Magic of Your Mind (Buffalo: Creative Education Foundation, 1981).

Rhodes, M. "An Analysis of Creativity," Phi Delta Kappan 42 (1961): 305-310.

Torrance, E. Paul. The Search for Satori and Creativity (Buffalo, N.Y.: Creative Education Foundation, 1979).

Von Oech, Roger. A Whack on the Side of the Head (New York: Warner Books, 1983).

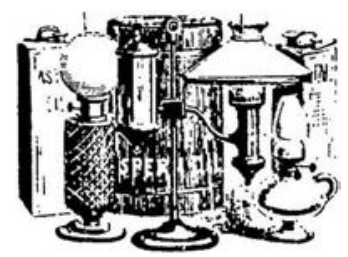

